\title{
The validity and reliability of the Turkish version of the Family Nutrition and Physical Activity screening tool
}

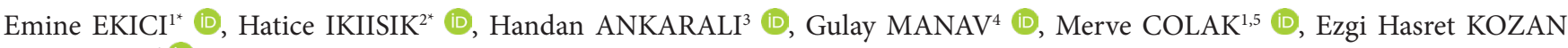 \\ CIKIRIKCI ${ }^{5,6}$ (i) \\ ${ }^{1}$ Department of Nursing, School of Health Sciences, Uskudar University, Istanbul, Turkey. \\ ${ }^{2}$ Department of Public Health, School of Medicine, Medeniyet University, Istanbul, Turkey. \\ ${ }^{3}$ Department of Bioistatistics, School of Medicine, Medeniyet University, Istanbul, Turkey. \\ ${ }^{4}$ Department of Pediatric Nursing, School of Health Sciences, Mugla Sitki Kocman University, Mugla, Turkey. \\ ${ }^{5}$ Department of Public Health, Cerrahpaşa Florence Nightingale School of Nursing, School, Istanbul, Turkey. \\ ${ }^{6}$ Department of Nursing, School of Health Sciences, Halic University, Istanbul, Turkey. \\ *Co-first authors. Both authors contributed equally to this paper.
}

Corresponding Author: Hatice IKIISIK

E-mail: hatice.ikiisik@medeniyet.edu.tr

Submitted: 02.01.2021 Accepted: 05.05.2021

\begin{abstract}
Objective: The aim of this study is to evaluate reliability and validity of the Turkish version of the Family Nutrition and Physical Activity (FNPA-TR) screening tool. This study was conducted as a methodological research. The data were collected between May and June 2018. The population of the study consisted of 1126 first and fourth grade students and their families in three primary public schools that represent three socioeconomic statuses (high, medium, low) in Istanbul, Turkey.

Materials and Methods: Data were collected from 727 students and their families with an introductory information form and the FNPA-TR screening tool. The validity of content scale was evaluated by comparing the relationship between FNPA-TR scores and answers given to non-scale questions measuring the eating behaviour. In this study, Cronbach's alpha, Kaiser-Meyer-Olkin, Varimax rotation and ICC coefficients statistical tests were used to measure validity and reliability.

Results: The Cronbach alpha coefficient for the internal consistency of the scale was 0.724 . The test-retest reliability coefficient of the scale had a medium to very high level that ranged from 0.422 to 0.925 . The Kaiser-Meyer-Olkin test result was found to be appropriate as 0.771 .

Conclusion: The study shows that the FNPA-TR scale is a valid and reliable measurement tool for the Turkish population.

Keywords: Family nutrition, Childhood obesity, Physical activity
\end{abstract}

\section{INTRODUCTION}

Obesity has turned out to be a multi-component disorder with the interaction of genes and the environment. The risk of obesity can be passed from generation to generation as a result of biological factors and/or behavioral factors. Children are known to inherit some characteristics from their families, such as socioeconomic status, cultural norms and behaviors, and family eating and physical activity behaviors, which are effective in the development of obesity in children [1].

Childhood obesity is a growing health problem for Turkish society as well as it is all over the world. The prevalence of obesity has increased threefold since 1975. The prevalence of overweight and obesity in all over the world, increased from 4\% in 1975 to
$18 \%$ in 2016 among children and adolescents aged between 5 and 19 [2]. There is an increase in childhood obesity in Turkey, as it is in the world. According to the Turkey Monitoring Growth in School-Age Children Survey in 2009 (TOCBI), overweight and obesity were $14.3 \%$ and $6.5 \%$ respectively in children aged between 6 and 10 [3].

Obesity has short and long-term effects on the health of children and adolescents. Compared to normal-weight children, obese children may suffer from absenteeism due to medical checks, health limitations, and diseases. Overweight and obese children

How to cite this article: Ekici E, Ikiisik H, Ankarali H, Manav G, Colak M, Kozan Cikirikci HE. The validity and reliability of the Turkish version of the Family Nutrition and Physical Activity screening tool. Marmara Med J 2021; 34(3):319-326. doi: 10.5472/marumj.1009125 
are more likely to become obese adults and have a higher risk of morbidity, disability, and premature death in adulthood [4-6].

School-age children have less autonomy in their choices of diet and physical activity. Parents have a direct impact on the home environment that may predispose the child to over-eating and inactivity [7]. The World Health Organization (WHO), World Health Assembly 2016 report of the Commission on Ending Childhood Obesity draws attention to the obesogenic environment, which is defined as an environment that supports excess energy intake and sedentary life. Several factors such as commercial factors, agricultural policies, food systems, access to healthy food, the necessary infrastructure for a healthy environment, and the family environment (parental nutrition knowledge and behavior, family economy, family eating behaviors) affect the obesogenic environment [8].

There is considerable research into obesogenic environmental components. Herbenick et al., evaluated the risk of obesity in children using the Family Nutrition and Physical Activity Screening Tool (FNPA) and anthropometric measurements. In the study, children's scores from the FNPA scale were found to increase as a result of training about the obesogenic environment given to school-age children [7]. In another study employing the FNPA scale, the FNPA was emphasized as a useful measurement tool for clinicians and programs aiming to change family behaviors and home environment in the fight against obesity [9]. In a qualitative study assessing the obesogenic environment, five main themes were attained to prevent obesity. Two of these were "positive parenting practices" and "gardens, parks, sports halls, and school meals". These themes demonstrated the effect of parental attitudes and activity to prevent obesity [10]. In another qualitative study conducted to determine parental perceptions of healthy living (especially nutrition and activation) and the content of interventions to be conducted in the future to prevent obesity in children, the nutrition experiences of families were found to be effective and difficult to change. In the same study, it was recommended that increasing self-efficacy in cases such as physical activity should be included in the strategies of the families involved in focus groups [11].

In the 2016 report of the WHO World Health Assembly, the Commission on Ending Childhood Obesity, five propositions were developed to end childhood obesity. These were listed as improving healthy food intake; improving physical activity; prenatal and pregnancy care; diet and physical activity in early childhood; and health, nutrition, and physical activity for school-age children [8].

Toruner et al., in their study comparing the status of measured body weight in children and the perceptions of parents, found that most parents did not notice obesity risk or obesity status in their children [12]. Since, families affect children's nutritional and physical activity behaviors and create an accessible social and physical environment, they are encouraged to be involved in the center of studies on the prevention of obesity in children $[13,14,15]$. For this reason, obesity screening strategies should also center on evaluating the family environment and their behaviors.
Assessing the nutritional and physical activity status of the family may be important in determining the causes of obesity in the child. We could not reach a reliable and valid screening tool to evaluate the nutrition and physical activity environment of the family in our country. This study aimed to adapt the FNPA Screening Tool to Turkish and evaluate its reliability and validity.

\section{MATERIALS and METHODS}

\section{Design}

A mix of probability sampling methods was used in this study, which was planned in the methodological type.

\section{Language Adaptation}

The items of the scale were translated into Turkish independently by three experts and then these texts were translated into English, the original language of the scale, separately by three different experts. Finally, a single translated text was created by comparing these translations by two researchers who have sufficient English language skills and are familiar with the measured concepts and the best Turkish translation form was determined for use in the study. To ensure the face validity of the translated text, the items were submitted to the opinions of three researchers with a background of the public health field, and the final version of the scale was obtained. The final form of the scale was named as "The Turkish Adaptation of the Family Nutrition and Physical Activity (FNPA-TR) Screening Tool”. A pilot study was conducted in an elementary school to see how the items of the FNPA-TR worked in the field. For this application, 15 families with children aged between 6 and 10, who were literate, who had no difficulty filling out the form due to health reasons, and who could speak Turkish were reached, and the actual implementation was started after necessary revisions were made. As a result of the pilot study, it was determined that answering the tool took 5-10 minutes on average and there was no problem in understanding the items.

The content validity of the scale was evaluated by academicians who had expertise on the subject. The explanatory factor analysis was employed for determining the item-factor relationship, and the confirmatory factor analysis was used for calculating the extent to which the items and the sub-dimensions explained the original structure of the scale. The time interval test-retest method was used for reliability analysis. Intra-class correlation coefficients (ICC) were found to range from 0.422 to 0.925 , and the scale was determined to vary between medium to high test-retest reliability. Cronbach's alpha coefficient related to the internal consistency of the scale was found to be 0.724 .

The permission from Ihmels et al, who developed FNPA screening tool was obtained through e-mail [13]. The institutional permission of Istanbul Provincial Directorate of National Education and the approval of Non-Interventional Research Ethics Committee from a university in Istanbul (issue: 23.03.2018/490) were obtained. The verbal consent of the children and the written consent of the parents were obtained. 


\section{Sample}

In the first stage of the sampling, schools were stratified according to their income levels, and among them, schools that were heterogeneous in terms of student profile and family profile were included in the study. The sample size was not calculated and it was aimed to reach all 1126 first and fourthgrade students and their families studying in three different primary schools with low, middle and high socioeconomic status in Uskudar, Istanbul.

There were 566 first-grade and 560 fourth-grade students in three selected public schools. Data forms were given to a total of 727 students and their families by researchers using face to face method, including 366 first-grade and 361 fourthgrade students, who agreed to participate in the study. Of the total students, $64.7 \%$ of the first-graders and $64.5 \%$ of the fourth-graders were contacted. The study was conducted with 591 students and their families who completed the data form and the questions were answered by the parents. Some of the questionnaire items were not responded by all participants, and therefore, \% values were calculated over the participants who responded to the items.

\section{Measures}

The data were collected using a questionnaire consisting of two parts:

Part 1: It consisted of 39 questions including the descriptive information of the participants and information on nutrition and physical activity (parent height, weight, age, marital status, educational status, occupation, social security, the longest residence, number of households, family monthly income, presence of chronic disease, etc.).

Part 2: The Turkish adapted Family Nutrition and Physical Activity ( FNPA-TR )Screening Tool : ABFA-TR is the Turkish version of the FNPA screening tool. The scale was developed in 2009 by Ihmels et al. [13].

This tool is an easy-to-use self-report scale designed to assess the family environment and behavioral factors that may cause the child to gain excess weight. The first version of the FNPA, which was created by identifying 10 main factors positively associated with overweight and obesity, was made up of a total of 21 questions. Then the number of items was reduced to 20 with the arrangements made in 2017 [16].

The conceptual framework of the scale consists of 10 subdimensions. Each item is rated on a four-point Likert type scale with options 1 = never / hardly ever, 2 = sometimes, 3 = often, 4 = usually / always. The scores that can be obtained from the scale vary between 1 and 80 points. The scale includes 10 subdimensions and two questions for each sub-dimension. Seven of the items $(3,4,5,7,10,13)$ were inversely coded.

The sub-dimensions are made of "Meals in the Family", "Family Eating Habits", "Food Choices", "Beverage Choices", "Limitation/ Rewarding", "Screen Time", "Healthy Environment", "Family Activity", "Child Activity", and "Family Planning / Sleep Pattern". The total score is calculated by summing the scores obtained from each sub-dimension. The total score is then used to interpret the physical activity and nutritional status of the family. A high total score obtained from the scale refers to a high-risk family environment and behaviors, while a low total score means a more positive family environment and behaviors.

The data were collected by the researchers between May and June 2018. A pilot study was conducted. To collect the data, the field team handed out the informed consent and the questionnaire involving the FNPA-TR scale in sealed envelope to the children to be taken to their parents. The responses received in the week following the day the envelopes were delivered were collected back in an envelope. Children's height and weight data were collected according to the statements of the families.

\section{Statistical Analysis}

The internal consistency of the scale and the subscales was calculated using Cronbach's alpha coefficient. Explanatory factor analysis was applied to 20 questions on the scale. The Kaiser-Meyer-Olkin test was used to determine whether the factor analysis was appropriate for the data structure. Besides, Bartlett's test was used for correlations between questions. Principal Components method was used to obtain factor loads, and the Varimax rotation method was used for identifying meaningful factor loads. ICC coefficients were calculated to find out the test-retest reliability. p-values less than 0.05 were considered statistically significant.

\section{RESULTS}

In this study the data from a total of 591 students is used including 294 (49.7\%) girls and 297 (50.3\%) boys from 3 different schools located in an Anatolian side district of Istanbul city, who were enrolled in the first $(\mathrm{n}=321,54.3 \%)$ and fourth ( $n=270,45.7 \%)$ grades and who filled out the questionnaire completely. Data were collected from primary schools from three different socioeconomic regions of the district. Of the families participating in the study, $115(19.5 \%)$ stated that their income was less than their expenses, 387 (65.5\%) reported their income as equivalent to their expenses, and 83 (14\%) said that their income was higher than their expenses. In addition, 506 of those who filled out the questionnaire forms were mothers (85.6\%) and $46(7.8 \%)$ were fathers.

The test-retest reliability of each item on the scale was analyzed using the intra-class correlation coefficient (ICC). The findings obtained from the analysis are given in Table I. The ICC coefficients were found to vary between 0.422 and 0.925 , which indicated test-retest reliability varying from a medium to a high level (Table I). 
Table I. Test-retest reliability results of scale items and sub-dimensions

\begin{tabular}{|c|c|c|c|c|}
\hline $\begin{array}{l}\text { Items and Sub- } \\
\text { Dimensions }\end{array}$ & ICC & $95 \%$ Confi & val for ICC & p \\
\hline Items & IUt & Lower & Upper & \\
\hline$A 1-1$ and $A 1-2$ & 0.846 & 0.731 & 0.912 & 0.001 \\
\hline A2-1 and A2-2 & 0.522 & 0.150 & 0.731 & 0.006 \\
\hline B3-1 and B3-2 & 0.777 & 0.611 & 0.872 & 0.001 \\
\hline B4-1 and B4-2 & 0.813 & 0.674 & 0.893 & 0.001 \\
\hline C5-1 and C5-2 & 0.614 & 0.327 & 0.779 & 0.001 \\
\hline C6-1 and C6-2 & 0.534 & 0.187 & 0.734 & 0.004 \\
\hline D7-1 and D7-2 & 0.545 & 0.206 & 0.740 & 0.003 \\
\hline D8-1 and D8-2 & 0.716 & 0.505 & 0.838 & 0.001 \\
\hline E9-1 and E9-2 & 0.556 & 0.225 & 0.746 & 0.002 \\
\hline E10-1 and E10-2 & 0.485 & 0.101 & 0.705 & 0.010 \\
\hline F11-1 and F11-2 & 0.422 & -0.009 & 0.669 & 0.027 \\
\hline F12-1 and F12-2 & 0.729 & 0.527 & 0.845 & 0.001 \\
\hline G13-1 and G13-2 & 0.623 & 0.338 & 0.786 & 0.001 \\
\hline G14-1 and G14-2 & 0.754 & 0.570 & 0.859 & 0.001 \\
\hline H15-1 and H15-2 & 0.799 & 0.649 & 0.885 & 0.001 \\
\hline $\mathrm{H} 16-1$ and H16-2 & 0.814 & 0.675 & 0.893 & 0.001 \\
\hline I17-1 and I17-2 & 0.768 & 0.595 & 0.867 & 0.001 \\
\hline I18-1 and I18-2 & 0.925 & 0.869 & 0.957 & 0.001 \\
\hline J19-1 and J19-2 & 0.778 & 0.613 & 0.873 & 0.001 \\
\hline $\mathrm{J} 20-1$ and $\mathrm{J} 20-2$ & 0.772 & 0.603 & 0.870 & 0.001 \\
\hline
\end{tabular}

$p<0.05$

As a result of the factor analysis applied to the 20 items on the scale, the Kaiser-Meyer-Olkin test result was found to be 0.771 . As this value was greater than 0.50 , the scale was determined to be appropriate for the factor analysis. Besides, the Sphericity test showed that the correlation matrix was not spherical ( $p$ $<0.0001)$. This result indicated that the correlations between the scale items were significant and appropriate for factor analysis. Furthermore, it was concluded that there was no need to eliminate any items from the scale because all diagonal elements of the anti-image matrix were greater than 0.50 . The factor analysis revealed that there were 7 factors with eigenvalues greater than 1. The scale exhibited a 7-factor structure explaining $58.1 \%$ of the variance in the sample. An analysis explaining $50-75 \%$ of the total variance is considered a valid analysis [17]. After obtaining factor loads, they were rotated using the Varimax rotation method, and 7 significant factors were obtained accordingly (Table II).

The first factor consisted of "Child Activity" with two items, "Family Activity" with two items, and the "How often does your family allocate time for physical activities (walking, running, etc.) item of the "Healthy Environment" sub-scale with two items. Its factor loads varied between 0.674 and 0.802 , and it explained $18.657 \%$ of the total variance.

The second factor consisted of "Meals in the Family" with two items and "How often does your child consume fruit and vegetables together with meals and as snacks (except for juice)?" item of the "Food Choices" with two items. Factor loads varied between 0.577 and 0.714 , and it explained $9.417 \%$ of the total variance.

"Sleep Pattern" sub-group made up the third factor that consisted of 2 items. Also, it explained $7.922 \%$ of the variance.

The "How often is fast-food consumed in your family?" item of the "Family Eating Habits" sub-group, the "How often does your family consume packaged fast-food? (frozen food, food heated in microwave, etc.)" item of the "Food Choices" sub-group, and the "How often does your child consume soda or sweetened beverages? (plain or fruit soda, cold tea, juice, energy drinks, etc.)" of the "Beverage Choices" sub-group made up the fourth factor. Factor loads were 0.645 and 0.768 and 0.555 . It explained $6.036 \%$ of the total variance.

The fifth factor consisted of the "How often does your family control your child's consumption of sugar, chips, and cookies?" item of the "Limitation/Rewarding" and the two items of the "Screen Time" sub-group. Factor loads were 0.686-0.638 and 0.571 . It explained $5.582 \%$ of the total variance.

As for the sixth factor, it was made up of the "How often does your child eat while watching TV (including meals and snacks)" item of the "Family Eating Habits" sub-group and the "How often does your child spend time on his computer, mobile devices, and game systems in his/her bedroom?" item of the "Healthy Environment" sub-group. Factor loads were 0.675 and 0.728. It explained $5.391 \%$ of the total variance.

Finally, the seventh factor was found to consist of the "How often does your child consume low-fat milk at meals or with snacks? ( $1 \%$ fat or skimmed milk, flavored milk, soy milk, almond milk, etc.)" item of the "Beverage Choices" sub-group and the "How often does your family use sweets, ice-cream, or other foods as a reward?" item of the "Limitation/Rewarding" sub-group. Factor loads were 0.627 and 0.576 and it explained the $5.066 \%$ of the variance. The factor structure of the items of the FNPA-TR, variance levels, and the item-total correlation values are presented in Table II. 
Table II. Factor structure of FNPA-TR scale, rotated factor loads, variance explanation shares and item total correlation values

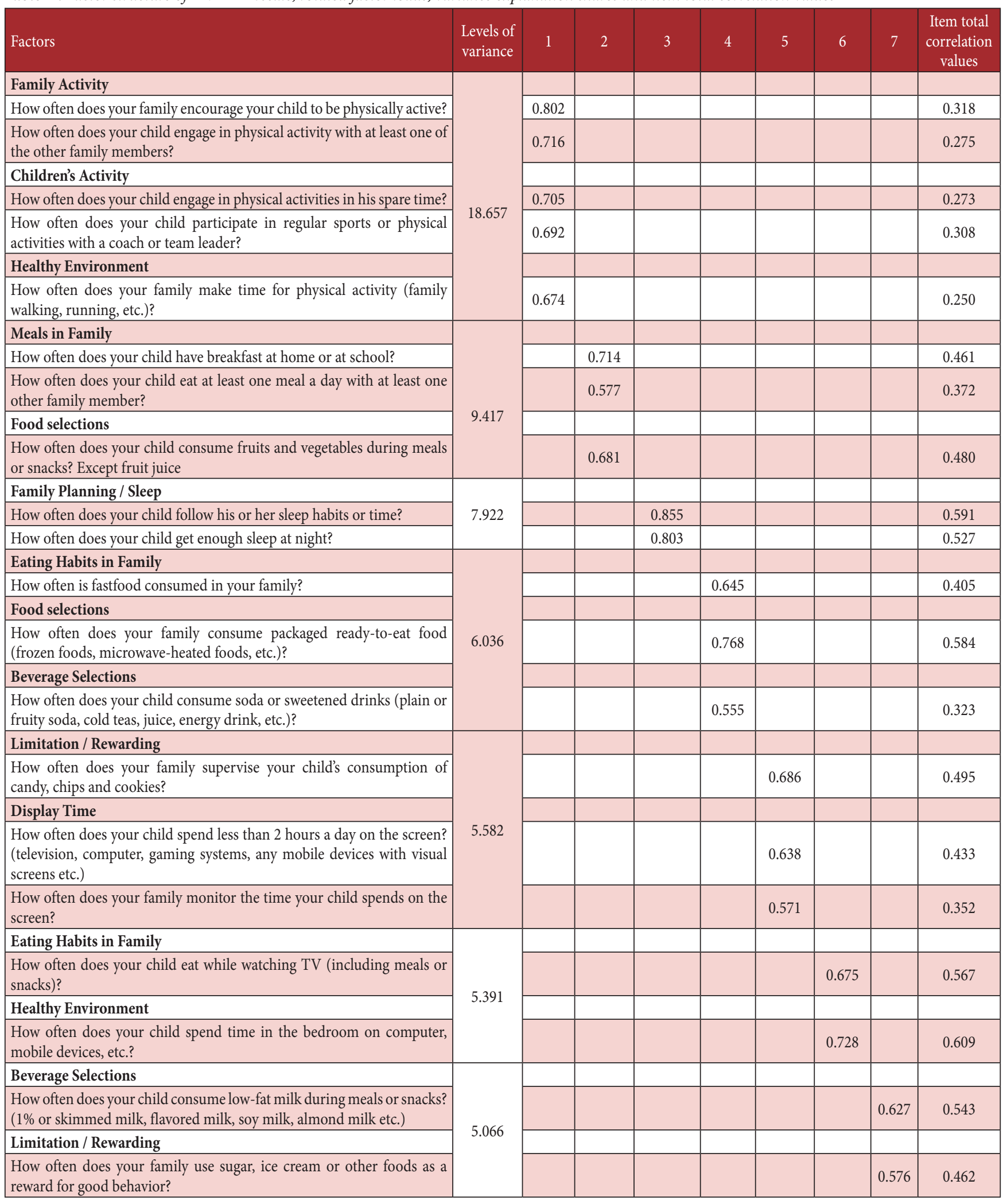


In the additional analyses conducted to evaluate the content validity, the relationships between the scale score and the answers given to questions which were not included in the questionnaire and which provided information about the feeding behavior of the family were investigated. When these relationships were evaluated, the mean score of those who had regular breakfast was significantly higher $(\mathrm{p}=0.001)$. The mean scale score was significantly higher in subjects who did not eat snacks and junk food ( $p=0.001)$. The mean scale score was found to be significantly higher in subjects who had regular meal hours $(p=0.001)$. There was no significant relationship between the number of meals a day and the scale score $(r=0.074, p=0.075)$. As the number of meals a week eaten together with the family increased, the scale score increased significantly as well $(\mathrm{r}=$ $0.167, \mathrm{p}=0.001$ ) (Table III).

Table III. Comparison of answers given to various questions in terms of total FNPA-TR scale score

\begin{tabular}{|c|c|c|c|}
\hline & n & Mean \pm SD & p \\
\hline \multicolumn{4}{|l|}{ Regular Breakfasts } \\
\hline Yes & 468 & $59.21 \pm 6.88$ & \multirow[t]{2}{*}{0.001} \\
\hline No & 106 & $53.18 \pm 5.54$ & \\
\hline \multicolumn{4}{|c|}{ Junk Food and Snacks Consumption } \\
\hline Yes & 148 & $55.61 \pm 6.77$ & \multirow{3}{*}{0.0001} \\
\hline No & 108 & $61.20 \pm 7.76$ & \\
\hline Sometimes & 321 & $58.12 \pm 6.48$ & \\
\hline \multicolumn{4}{|c|}{ Do you eat meals at regular times? } \\
\hline Yes & 395 & $59.72 \pm 6.92$ & \multirow{3}{*}{0.001} \\
\hline No & 40 & $51.90 \pm 6.17$ & \\
\hline Sometimes & 143 & $55.19 \pm 5.62$ & \\
\hline \multicolumn{4}{|l|}{ Gender } \\
\hline Female & 294 & $58.44 \pm 6.43$ & \multirow{2}{*}{0.230} \\
\hline Male & 297 & $57.74 \pm 7.70$ & \\
\hline \multicolumn{4}{|l|}{ Grade } \\
\hline 1st Grade & 321 & $57.19 \pm 7.36$ & \multirow{2}{*}{0.001} \\
\hline 4th Grade & 270 & $59.15 \pm 6.62$ & \\
\hline \multicolumn{4}{|l|}{ Income } \\
\hline Income is less than expense & 115 & $56.13 \pm 7.37$ & \multirow{3}{*}{0.001} \\
\hline Income is equivalent to expense & 387 & $58.51 \pm 6.92$ & \\
\hline Income is more than expense & 83 & $58.63 \pm 6.88$ & \\
\hline
\end{tabular}

\section{DISCUSSION}

This study investigated the validity and reliability of the Turkish adapted version of the "Family Nutrition and Physical Activity Screening Tool" (FNPA) [15], which was developed to evaluate the home environment and family nutrition and physical activity practices and behaviors that may be associated with childhood overweight and obesity. The study supported the original FNPA findings

The time interval test-retest method was used for reliability analyses. The intra-class correlation coefficients (ICC) were found to range between 0.422 and 0.925 and have a medium to very high test-retest reliability. Cronbach's alpha coefficient related to the internal consistency of the scale was found to be 0.724. Cronbach Alpha value between 0.71-0.91 indicates that the scale has a good level of reliability [18].

Explanatory factor analysis was employed for determining the item-factor relationship and confirmatory factor analysis was used for calculating the extent to which the items and the factors in the scale explained the original construct of the scale. As a result of the factor analysis conducted to determine the factor construct of the scale, the scale was determined to consist of 7 factors. The item-factor distribution was as follows. Factor 1: child activity $(1,2)$, family activity $(1,2)$, and healthy environment (2); factor 2: meals in the family $(1,2)$, and food choices (2); factor 3 : sleep pattern $(1,2)$; factor 4 : family eating habits (2), food choices (1), and beverage choices (1); factor 5: limitation / rewarding (1) and screen time $(1,2)$; factor 6: family eating habits (1) and healthy environment (1); factor 7: beverage choices (2) and restriction / rewarding (1) (Table IV). In factor analyses conducted to examine the psychometric properties of the original FNPA scale, 7 factors met the minimum eigenvalue criterion of 1.0 .

A significant correlation was found between the total scale score and the BMI of the child (-0.03), the mother $(r=-0.15)$, and the father $(r=-0.02)$. This weak correlation was likely to stem from the large sample size. Similar relationship between the BMI of the child and FNPA scores was observed in the original validity study $(\mathrm{r}=.10 .17, \mathrm{p}<0.01)$ and in another study conducted on different age groups (first-grade students: $r=-0.17$; tenth-grade students: $r=-0.15)[12,14]$. Another study showed a negative correlation between age and gender-adjusted FNPA scores and $\mathrm{BMI}, \mathrm{BMI}$ at the 95 th percent, $\mathrm{BF} \%$, and all fat prevention measures, including the waist [9].

In the present study, a significant relationship was found between the BMI of the parents and the BMI of the child. Studies show that the environment shared by individuals and their behaviors as well as genetic factors affect the relationship between the BMI of the parents and the child [19-21]. This finding on the relationship between parent BMI and child BMI emphasizes the need for a family-based approach to the prevention of childhood obesity. There was also a significant relationship between the BMI of the mother and the FNPA total score. Parents' behaviors related to nutrition and physical activity are positively related to their own weight management as well as the obesogenic environment in the family and the nutritional quality and/or weight status of children [9, 14, 22, 23]. Williams et al., also found a relationship between the parent's BMI and the family FNPA score and reported that a large proportion of families with a low or normal weight parent had high FNPA scores, and a smaller proportion of families with overweight or obese parents had high FNPA scores [24].

A large portion of studies on childhood obesity has reported an inverse relationship between socioeconomic status (SES) and childhood obesity $[25,26]$. However, there are studies showing that the relationship between SES and obesity is directly proportional. These studies have mentioned that in 
addition to SES, factors such as ethnic inequalities, parental education, family income, and changes occurring over time may be effective on obesity $[27,28]$. In the present study, the mean BMI of children was observed to be higher in schools with high and middle socioeconomic levels compared to the low ones. In addition, the mean child BMI was higher in families reporting higher income than their expenses.

Evaluations of the mean BMI should include not only SES or family income but also environmental, biological, and sociocultural factors. As studies on inequalities addressing childhood obesity have focused on individual behaviors, maternal education, SES, family behaviors, sedentary behaviors, snacks between meals, eating habits such as consumption of fast-food and sweetened drinks, physical activity, and breastfeeding, the potential effects of social environments on obesity are still unclear $[7,10]$. Although, ethnicity is stated to have a role in this difference $[11,29]$, this study did not include any elements of ethnicity.

The evaluation of cultural and living conditions suggests that children of families with high income and SES have no financial barriers to access to unhealthy snacks such as fast-food, chips, and sweet foods, as well as access to high-tech technology such as television, computer games, and telephones; consequently, these conditions result in decreased physical activity, and they are thought to be effective in the emergence of these results.

The results of the study showed that the higher the socioeconomic status of the schools was, the higher their FNPA total scores were. Also, the mean scale score was found to be significantly higher in those who had regular breakfast, who did not eat junk food and snacks, and who had regular meals. Regular breakfast and eating regular meals were reported to be factors that decreased the rate of obesity in children.

The obesity epidemic makes it essential to improve our understanding of the effect of food environments on children. As healthy habit formation at an early age is essential in primary prevention of obesity, it is necessary to control nutritional behaviours, familial factors and physical activity levels. This tool can be used to evaluate environmental factors affecting obesity to create programs to prevent obesity, as well as other anthropometric measurements such as waist-to-height ratio, BMI and waist circumference.

Studies are carried out to increase adequate and balanced nutrition and physical activity in the prevention of childhood obesity. Parents have a direct impact on the home environment which may predispose their children to excessive food intake, excess energy intake, and inactivity. It is important to provide a healthy family environment that improves eating behaviors and physical activities of children and to take part in their lives as the right role model. Developing and using measurement tools related to the family environment as an element of the obesogenic environment is among important steps to be taken to fight obesity. In conclusion, the Turkish version of the FNPA is a valid and reliable screening tool and it captures various aspects of the home environment rather than just physical activity or diet. We think FNPA will meet the need for a reliable obesogenic environmental assessment in our country.

\section{Compliance with Ethical Standards}

Ethical Approval: The study was approved by Uskudar Universty, Non-Interventional Research Ethics Committee (issue: 23.03.2018/490). The institutional permission was obtained from the Istanbul Provincial Directorate of National Education. The verbal consent of the children and the written consent of the parents were obtained.

Financial Support: The authors have no relevant financial information to disclose.

Conflict of interest: The authors declare that they have no conflict of interest to declare

Authors' contribution: E.E and H.I. conceived of the presented idea. E.E., H.I., M.C. and E.H.K. developed the theory and collected research data. H.A. verified the analytical methods. All authors discussed the results and approved the final version of the article.

\section{REFERENCES}

[1] Maziak W, Ward KD, Stockton MB. Childhood obesity: are we missing the big picture? Obes Rev. 2008 Jan;9(1):35-42. doi: 10.1111/j.1467-789X.2007.00376.x.

[2] World Health Organization. Obesity and overweight. Key facts. 2018. Available from: http://www.who.int/mediacentre/ factsheets/fs311/en/. (Accessed: 21 March 2020)

[3] Monitoring the Growth of School Age Children (6-10 Age Group) in Turkey (TOÇBI) Project Research Report, Ministry of Health, General Directorate of Basic Health Services, Hacettepe University Faculty of Health Sciences, Department of Nutrition and Dietetics, Ministry of National Education, Ministry of Health Publication No: 834;2011

[4] Public Health England, Chilhood Obesity Health Risks. 2013. Available from: http://www.noo.org.uk/NOO_about_obesity/ obesity_and_health/health_risk_child. (Accessed: 20 May 2020)

[5] Guidelines For Adolescent Nutrition Services. Stang J, Story M (eds). 2015. Available from: http://www.epi.umn.edu/let/ pubs/img/adol_preface_materials.pdf. (Accessed: September, 2020)

[6] Kansra AR, Lakkunarajah S, Jay MS. Childhood and adolescent obesity: a review. Front Pediatr 2021;12;8:581461. doi: 10.3389/fped.2020.581461.

[7] Herbenick SK, James K Milton J, Cannon D. Effects of family nutrition and physical activity screening for obesity risk in school-age children. J Spec Pediatr Nurs 2018 ; 23:e12229. doi: 10.1111/jspn.12229

[8] World Health Organization. Report Of The Commision On Ending Childhood Obesity. 2016. Availableform: http://apps. who.int/iris/bitstream/10665/204176/1/978.924.1510066_ eng.pdf. (Accessed: September, 2020) 
[9] Tucker JM, Howard K, Guseman EH, Yee KE, Saturley H, Eisenmann JC. Association between the Family Nutrition and Physical Activity Screening Tool and obesity severity in youth referred to weight management. Obes Res Clin Pract. 2017 May-Jun;11(3):268-275. doi: 10.1016/j.orcp.2016.09.007.

[10] Stang J, Bonilla Z. Factors Affecting Nutrition and Physical Activity Behaviors of Hispanic Families With Young Children: Implications for Obesity Policies and Programs. J Nutr Educ Behav. 2018 Nov-Dec;50(10):959-967. doi: 10.1016/j. jneb.2017.08.005.

[11] Taverno Ross SE, Clennin MN, Dowda M, Colabianchi C, Pate RR. Stepping It Up: Walking Behaviors in Children Transitioning from 5th to 7th Grade. Int. J. Environ. Res. Public Health. 2018; Feb 3;15(2). pii: E262. doi: 10.3390/ ijerph15020262

[12] Toruner EK, Ayaz S, Savaser S. Parents' perceptions about weights of their children. Hemar-G. 2014; 16(2): 11-20.

[13] Ihmels MA, Welk GJ, Eisenmann JC, Nusser SM. Development and preliminary validation of a Family Nutrition and Physical Activity (FNPA) screening tool. Int J Behav Nutr Phys Act. 2009; 6:14. doi: 10.1186/1479-5868-6-14

[14] Bailey-Davis L, Peyer KL, Fang Y, Kim JK, Welk GJ. Effects of Enhancing School-Based Body Mass Index Screening Reports with Parent Education on Report Utility and Parental Intent To Modify Obesity Risk Factors. Child Obes. 2017;13(2):164171. doi:10.1089/chi.2016.0177

[15] Karnik S, Kanekar A. Childhood obesity: a global public health crisis. Int J Prev Med 2012;3:1-7..

[16] Peyer KL, Welk GJ. Construct Validity of an Obesity Risk Screening Tool in Two Age Groups. Int J Environ Res Public Health. 2017 Apr 14;14(4):419. doi: 10.3390/ijerph14040419.

[17] Yurdalan SU, Sengir N, Isci E. Turkish Validity and Reliability of DREEM Questionnaire used in assessment of learning environment in physiotherapy and rehabilitation undergraduate education. JHMN 2020; 78: 8-17. doi: 10.7176/ JHMN/78-02

[18] Taber KS. The Use of Cronbach's Alpha when developing and reporting research instruments in science education. Res Sci Educ 2018; 48: 1273-1296. https://doi.org/10.1007/ s11165.016.9602-2

[19] Wardle J, Camell S, Haworth CM, Plomin R. Evidence for a strong genetic influence on childhood adiposity despite the force of the obesogenic environment. Am J Clin Nutr. 2008 Feb;87(2):398-404. doi: 10.1093/ajcn/87.2.398

[20] Herbert A, Gerry NP, McQueen MB et al. A common genetic variant is associated with adult and childhood obesity. Science. 2006 Apr;14;312(5771):279-83. doi: 10.1126/science.1124779

[21] Maes HH, Neale MC, Eaves LJ. Genetic and environmental factors in relative body weight and human adiposity. Behav Genet. 1997 Jul;27(4):325-51. doi: 10.1023/a:102.563.5913927

[22] Couch SC, Glanz K, Zhou C, Sallis JF, Saelens BE. Home food environment in relation to children's diet quality and weight status. J Acad Nutr Diet. 2014;114(10):1569-1579.e1. doi:10.1016/j.jand.2014.05.015

[23] Conroy SM, Shariff-Marco S, Yang J et al. Characterizing the neighborhood obesogenic environment in the Multiethnic Cohort: a multi-level infrastructure for cancer health disparities research. Cancer Causes Control. 2018 Jan;29(1):167-183. doi: 10.1007/s10552.017.0980-1.

[24] Williams JE, Helsel B, Griffin SF, Liang J. Associations between parental BMI and the family nutrition and physical activity environment in a community sample, J Community Health 2017; 42:1233-1239 doi: 10.1007/s10900.017.0375-y

[25] Small M, Bailey-Davis L, Morgan N, Maggs J. Changes in eating and physical activity behaviors across seven semesters of college: living on or off campus matters. Health Educ Behav. 2013;40(4):435-441. doi:10.1177/109.019.8112467801

[26] Scharoun-Lee M, Kaufman JS, Popkin BM, et al. Obesity, race/ethnicity and life course socioeconomic status across the transition from adolescence to adulthood. JECH 2009;63:133139. doi: 10.1136/jech.2008.075721

[27] Gordon-Larsen P, Adair LS, Popkin BM. The relationship of ethnicity, socioeconomic factors, and overweight in US adolescents. Obes Res. 2003;11(1):121-129. doi: 10.1038/ oby. 2003.20

[28] Wang Y, Zhang Q. Are American children and adolescents of low socioeconomic status at increased risk of obesity? Changes in the association between overweight and family income between 1971 and 2002. Am J Clin Nutr. 2006 Oct;84(4):70716. doi: 10.1093/ajcn/84.4.707

[29] Rossen LM Neighbourhood economic deprivation explains racial/ethnic disparities in overweight and obesity among children and adolescents in the USA. JECH 2014;68:123-129. doi: $10.1136 /$ jech-2012-202245 